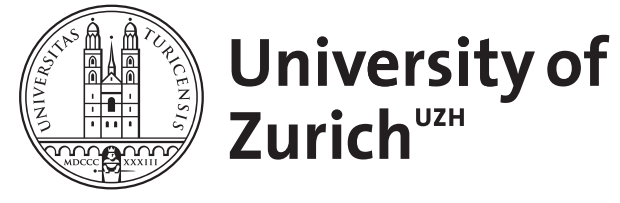
Archive

University of Zurich

University Library

Strickhofstrasse 39

CH-8057 Zurich

www.zora.uzh.ch

Year: 2012

The impact of hypoxia on cells, mice, and men

Gassmann, Max ; Ogunshola, Omolara O ; Tissot van Patot, Martha C

DOI: https://doi.org/10.1089/ham.2011.1108

Posted at the Zurich Open Repository and Archive, University of Zurich

ZORA URL: https://doi.org/10.5167/uzh-62404

Journal Article

Published Version

Originally published at:

Gassmann, Max; Ogunshola, Omolara O; Tissot van Patot, Martha C (2012). The impact of hypoxia on cells, mice, and men. High Altitude Medicine and Biology, 13(1):63-64.

DOI: https://doi.org/10.1089/ham.2011.1108 


\title{
The Impact of Hypoxia on Cells, Mice, and Men
}

\author{
Max Gassmann, Omolara O. Ogunshola, and Martha Tissot van Patot
}

$\mathbf{T}$ HIS Is A REPORT OF THE HypoxiaNet meeting that took place at Monte Verità in Ascona, Switzerland, October 9 14,2011 . It was the third in a series of meetings held at Monte Verità designed to bring together biomedical scientists and clinician researchers who study the role of hypoxia in diverse fields. Initially, investigations into the role of hypoxia in the etiology of disease were limited to the pulmonary field particularly in relation to the effects of rapid ascent to and residence at high altitude. Over the past 20 years, it has become increasingly apparent that hypoxia plays a significant role in an enormous variety of diseases, from pregnancy disorders to formation of tumors. This meeting series was the first dedicated to bringing together basic research scientists, physiologists and clinicians from widely differing backgrounds to compare and contrast the role of hypoxia in specific cellular and animal models as well as different human disorders and diseases.

These close-knit meetings are designed such that the maximum of 100 attendees, including speakers, attend a single series of lectures throughout the day. All participants stay at the beautiful and historic Monte Verità Hotel and Conference Center, taking meals together at large community tables. This arrangement provides extensive opportunities for discussion and developing unique collaborations. The attendees embraced the spirit of the meeting with molecular biologists attending presentations on high altitude-induced disease and clinical oncologists attending sessions on the role of mitochondria in hypoxic cells.

The current meeting was divided into seminars focusing on areas such as the roles of specific molecular pathways in hypoxia (HIFs, PHDs, and erythropoietin), physiological pathways (mitochondrial respiration, ventilation), specific organ responses to hypoxia (eye, placenta, gut) and clinical repercussions (exercise, high altitude cerebral and pulmonary edemas, adaptation). Despite speakers from a highly varied number of scientific fields, it became clear that there was a thread of important commonalities throughout the research presentations, tying data together into a global view of hypoxic response.

As such, the keynote lecture by Dr. William G. Kaelin, M.D., describing new cancer therapies developed from recent understanding of the molecular pathways in the mammalian oxygen sensing pathways, focusing primarily on HIF prolyl hydroxylases (PHDs), proved prophetic and provided a great foundation for the start of the conference. PHD involvement was demonstrated throughout the meeting in a wide-ranging list of diseases including, cardiomyopathy (Katschinski, DM), cardiac ischemic reperfusion injury (Koivunen, P), inflammatory bowel disease (Taylor, C), and Tibetan adaptation to high altitude residence (Prchal, J).

Mitochondria activity was another commonality among hypoxia research presentations including generation of reactive oxygen species (Schumaker, P), hydrogen sulfide production/carotid body sensitivity (Kemp, P), placental development at high altitude (Tissot van Patot, M), adaptation to high altitude ascent (Gelfi, C), exercise (Gnaiger,E), body weight control (Aragonés, J), ischemia-reperfusion lung injury (Weissmann, N), breast cancer (Gorr, T), and ischemic cardioprotection (Koivunen, P). Mitochondrial response to hypoxia seems to be common denominator in numerous hypoxia-mediated conditions potentially providing another strong target for developing therapeutic interventions.

The more recently defined role of epigenetics in response to hypoxia was prominently discussed in a variety of conditions as well. Data were presented implicating hypoxia in altering epigenetics via chromatin remodeling (Poellinger, L), miRNAs (Gay, S), and DNA methylation (Sartori, C) in cancer, rheumatoid arthritis, and pulmonary hypertension, respectively. The potential role for the amazing capacity of epigenetics to be altered in a single generation was then further discussed in presentations focusing on adaptations in second generations at altitude, adaptation of llamas (Llanos, A), and population adaptation in Tibetans and Andeans (Prchal, J). Subsequently, evidence of hypoxia-induced rough endoplasmic reticular stress, modifications in protein synthesis and/or effects on cellular proliferation and migration was presented in association with metastasis (Johnson, R), retinal development (Grimm C) and disease (Bösch, M), as well as placental adaptation to high altitude (Burton, G). Interestingly, exposure of healthy volunteers to high altitude (Capanna Margherita) was shown to cause ulceration in the stomach (Lutz, $\mathrm{T}$; Goetze, O). Finally, Jerry Dempsey reviewed the causes and consequences of hypoxic ventilatory acclimatization.

Recent evidence that erythropoietin (Epo) has multiple non-erythroid functions was expanded by data implicating Epo in exercise performance by hematological and nonhematological functions (Gassmann, M), improved cognition (Ehrenreich, H) and mood disorders (Miskowiak, K), retinal function $(\operatorname{Rex}, \mathrm{T})$, neonatal ventilation (Soliz, J), and protection from ventilator-induced lung injury (Swenson, E). An excellent debate of the mode of action of Epo protection and physiological effects was also initiated in these sessions. 
Other similarities between hypoxic research areas include iron homeostasis implicated in kidney fibrosis (Haase, V.) and skeletal muscle response (Gelfi, C), nitric oxide implicated in ischemic cardioprotection (Koivunen, P), differential activation of HIF-1 and HIF-2 (Johnson, R), and control of Sglutathionylation (Bogdanova, A).

An excellent set of sessions on response to high altitude provided a valuable context in which to view the variety of hypoxia-induced pathways coming together for a global physiologic/pathophysiologic response. There were lively debates about the role of Epo in exercise, the limitations of exercise at altitude and the role of doping in climbers. The questions surrounding the doping issue included: is prevention or treatment of altitude illness doping and what constitutes doping in elite climbers? (chair: Oelz, O). There was also an interesting discussion session in which a consensus on the prevention and treatment of high altitude pulmonary edema (HAPE) was sought (chair: Maggiorini, M). Data on exercise and pulmonary capacity, pulmonary disease and altitude ascent, HAPE susceptibility and a variety of presentations on treatments including ascent rate recommendations, oxygen, acetazolamide, phosphodiesterase 5 inhibitors, steroids and beta- 3 agonist formed the context in which a consensus on HAPE was based.

Because of the meeting environment, the excellent science, the novel mixture of scientific fields all surrounding hypoxia, the organization of the workshops, and the excellent discussions during the presentations, the meeting was an unparalleled success. The meeting successfully achieved the projected goals of bringing together scientists to discuss the state of the art in our understanding of the effects of hypoxia on the body, from development to aging as well as during pathophysiology processes, and developing new directions for future exploration.

Address correspondence to: Professor Max Gassmann Institute of Veterinary Physiology and Zurich Center for Integrative

Human Physiology

University of Zurich

Winterthurerstr. 260

Zurich 8057

Switzerland

E-mail: max@access.uzh.ch 